\title{
Potential synergism of Bim with p53 in mice with Myc-induced lymphoma in a mouse lymphoma model
}

\author{
QINGJUAN SHANG ${ }^{1}$, DIANLIANG ZHANG ${ }^{2}$, CHUNBAO GUO ${ }^{3}$, \\ QIU LIN ${ }^{3}$, ZHENHUA GUO ${ }^{3}$ AND CHUN DENG ${ }^{3}$ \\ ${ }^{1}$ Department of Pathology, Linyi Municipal Hospital, Linyi, Shandong; ${ }^{2}$ Department of Surgery, \\ the Affiliated Hospital of Medical College, Qingdao University, Qingdao; ${ }^{3}$ Laboratory of Surgery, \\ Children's Hospital of Chongqing Medical University, Chongqing, P.R. China
}

Received September 8,2011; Accepted October 24, 2011

DOI: $10.3892 / \mathrm{mmr} .2012 .844$

\begin{abstract}
We report that Bim has an apoptotic function through Bax/mitochondrial apoptosis signaling in the p53-independent mode, which is somewhat additive to the effects of p53. Bim-deficient mouse embryonic fibroblast (MEF) cells were resistant to the apoptotic effects of Myc, while loss of Bim accelerated lymphoma development. Furthermore, Bim was overexpressed at the same frequency in Myc-initiated lymphomas, irrespective of p53 status, suggesting that Bim resides in a pathway separate from p53. Loss of Bim further augmented resistance to apoptosis in $\mathrm{p} 53^{-/-}$MEFs. Mice with p53 knockdown exhibited exacerbated malignancies in the absence of Bim. The combined loss of these proteins promoted more severe spontaneous tumorigenesis. Thus, Myc-induced apoptotic signals through Bim and p53 must bifurcate to activate Bax, suggesting that the activation of Bim and p53 significantly contribute to apoptosis. Our results, therefore, establish that p53 and Bim are effective key initiators of apoptosis in lymphoma cells, particularly when combined.
\end{abstract}

\section{Introduction}

Over $30 \%$ of human tumors highly express the Myc proto-oncogene. Paradoxically, Myc plays a crucial role in cell proliferation and apoptosis $(1,2)$. Aside from its cell proliferation activities, Myc is capable of inducing or sensitizing cells to apoptosis by inducing cytochrome $\mathrm{c}$ release that depends on caspase activation (3). Impaired apoptosis of Myc is linked to lymphomagenesis in mice. Any mutations that prevent cells from undergoing apoptosis may be oncogenic, a phenomenon that is well documented but incompletely understood (4-6).

Correspondence to: Dr Chunbao Guo, Laboratory of Surgery, Children's Hospital of Chongqing Medical University, 136 Zhongshan 2nd Rd. Chongqing 400014, P.R. China

E-mail: gchunbao@yahoo.com.cn

*Contributed equally

Key words: Myc, Bim, p53, lymphoma, BH3-only proteins
It has been proposed that a group of $\mathrm{BH} 3$-only proteins may be involved during apoptosis in cells and this depend on oncogenic Myc. The BH3-only proapoptotic proteins are proven to be the major physiological antagonists of Bcl-2, which may mediate Myc-induced apoptosis of lymphoma cells (7-9). During Myc-induced lymphomagenesis, the one protein most consistently implicated as cooperating with Myc is p53; however, apoptosis is attenuated but not completely suppressed when the p53 pathway is inactivated. While the significance of Bim and other genes has been recognized (10-12) during Myc-involved lymphomagenesis, independently of p53 (13), it is unclear as to whether these genes are absolutely required for neoplastic growth or are merely selected during the late stages of tumor progression (14). We hypothesized that the BH3-only members, particularly Bim, play a crucial role following Myc deregulated expression and might be potential novel apoptotic induction targets for future tumor intervention.

To determine whether Myc overexpression is capable of targeting Bim to promote tumorigenesis in mice, we used a system for rapidly generating tissue-specific transgenic mice to study Bim's role in Myc-induced apoptosis in lymphomagenesis. Notably, the additional loss of Bim (p53 independent) over p53 deficiency elevated the rate of spontaneous tumor development and resisted against drug-induced cell death, confirming the intrinsic tumor-suppressor potential of these proteins. Thus, constraining the cellular apoptotic program by genetic targeting of the BH3-only proapoptotic proteins, such as Bim, not only controls sensitivity of lymphoma cells to Myc but also determines therapeutic outcome.

\section{Materials and methods}

Primary mouse embryonic fibroblast (MEF) harvest and retroviral infection in vitro. Primary MEFs were obtained from E13.5 embryos. Retroviral infections were carried out using murine stem cell virus (MSCV) vectors encoding green fluorescent protein (GFP) 3' of an internal ribosomal entry site (IRES) of a bicistronic message. MSCV-GFP and MSCV-GFP-WT MYC were kindly provided by S.W. Lowe. Murine Bim was cloned into pMSCV-IRES-GFP as a 761-bp BglII-EcoRI fragment (MSCV-Bim). Retroviral-mediated gene transfer was performed using Phoenix packaging cells, 
as previously described (1). Aliquots of harvested MEFs ( $3 \times 10^{6}$ cells) were infected with MSCV-GFP, MSCV-GFP-WT MYC and MSCV-Bim through coculturing with high titer retroviral supernatants. Cocultures were incubated four times by spinoculation at $600 \mathrm{x} \mathrm{g}$ for $10 \mathrm{~min}$ in $3 \mathrm{ml}$ of retroviral supernatant every 6-8 h. Growth factors were included in the suspension culture at the following concentrations: $20 \mathrm{ng} / \mathrm{ml}$ murine IL-3 (R\&D Systems, Minneapolis, MN, USA), $50 \mathrm{ng} / \mathrm{ml}$ human IL-6 (R\&D Systems), $50 \mathrm{ng} / \mathrm{ml}$ murine stem cell factor (SCF) (R\&D Systems) and $2 \mu \mathrm{g} / \mathrm{ml}$ Polybrene (Sigma, St. Louis, MO, USA). A total of $24 \mathrm{~h}$ following the last infection, the fraction of GFP-expressing cells was measured by flow cytometry (FACScalibur, Beckton Dickinson).

Bone marrow reconstitution, lymphoma monitoring and analysis. The recipient $\mathrm{C} 57 \mathrm{BL} / 6$ inbred strain of mice (6-8 weeks old) was lethally irradiated with a 10 Gy-dose of total body irradiation (TBI) $(0.8 \mathrm{~Gy} / \mathrm{min})$ using a $60 \mathrm{Co}-\gamma$ ray source. Aliquots of $1-2 \times 10^{6}$ viable nucleated fetal liver cells in $0.5 \mathrm{ml}$ or less of phosphate-buffered saline (PBS) were collected in 1-ml tuberculin syringes and infused slowly through the central tail vein of the recipients, as described previously (18). Mice were housed on autoclaved bedding in air-filtered cages and received neomycin-containing drinking water. Radiation controls were simultaneously prepared to confirm the adequacy of the lethal irradiation dose. Animals were euthanized and monitored for illness by lymph node (LN) palpation and were monitored for overall morbidity. The occurrence of well-palpable peripheral LN enlargements defined the onset of lymphoma. Overall survival was defined at the time from stem cell reconstitution until the animal reached a terminal stage. Statistical evaluation of tumor onset is based on the log-rank test for comparison of the Kaplan-Meier event-time format. Reconstituted animals, harboring control and p53/- lymphomas, were, in some cases, subjected to whole-body fluorescence imaging. In certain cases, animals were also sacrificied at predetermined times by cervical dislocation. Spleens, lung, liver and tumor tissue were removed en bloc at necropsy, exsanguinated with PBS washing and placed on dry ice immediately in order to subject tissues to staining with hematoxylin and eosin (H\&E). This method enables investigators to evaluate apoptotic nuclear morphology and the invasiveness of lymphoma cells into visceral organs. Lymphoma tissues were either fixed for histopathological evaluation or processed to single-cell suspensions for subsequent assays and cell culture.

Isolation and screening of lymphoma cells with Bim knockdown or transduction. Lymphoma-bearing mice were sacrificed by $\mathrm{CO}_{2}$ euthanasia. LNs were dissected, minced in PBS and filtered through a $35-\mu$ m nylon mesh. Single cell suspensions of freshly harvested lymphomas were incubated on an irradiated (30 Gy) feeder layer $\left(10^{6} \mathrm{NIH}-3 \mathrm{~T} 3 \mathrm{cells} / 2.4-\mathrm{cm}\right.$ plate) at $37^{\circ} \mathrm{C}$ in a humidified atmosphere containing $5 \% \mathrm{CO}_{2}$ and immunophenotyped by flow cytometry using antibodies directed against B220 and immunoglobulin M (IgM) (Pharmingen, Franklin Lakes, NJ USA). The high-purity small interfering RNAs (siRNAs) SignalSilence ${ }^{\circledR}$ Bim siRNA kit (Cell Signaling Technology, MA, USA) and control siRNA were purchased from Upstate (Charlottesville, VA,
USA). The lipid encapsulated SMARTpool ${ }^{\mathrm{TM}}$ and control were used for transfection. At the time of transfection, the cell density was 70-90\% confluent, or at a density of approximately $1 \times 10^{5}$ cells $/ \mathrm{ml}$. We transfected the cells using siRNAs with the siIMPORTER ${ }^{\mathrm{TM}}$ Transfection Reagent (Upstate), following the manufacturer's instructions and taking measures to test and optimize the conditions bestsuited for the cell line or culture of choice. To stably knock down the expression of Bim, we used the BLOCK-iT Inducible H1 Lentiviral RNAi System ${ }^{\mathrm{TM}}$, which was purchased from Invitrogen, according to the manufacturer's instructions (Invitrogen, CA, USA). The insert strand sequences of the short hairpin RNA (shRNA)-encoding oligonucleotides were 5'-GATCCCCCGTATGCGG AATACTTTGATTCAAGAGATCGAAGTATTCCGCGTA CGTTTTTC-3' for control (luciferase) and 5'-GATCCCCGA CTGAGAAGGTAGATAATTTTCAAGAGAAATTGTCTA CCTTCTCGGTCTTTTTC-3' for Bim. We transfected the lymphoma cells with $10 \mu \mathrm{l}$ rat Bim-shRNA virus per well of a 12-well plate in the presence of polybrene (Fisher Scientific, Tustin, CA, USA) for adherent cells, following the manufacturer's instructions and taking measures to test and optimize the conditions best-suited for the cell line or culture of choice. Gene silencing effects were evaluated by Western blot analysis. For the construction of the tetracyclineregulated gene expression vectors expressing Bim, the coding region of mouse Bim was PCR-amplified and subcloned into pTHE vector, resulting in pTHE-Bim. All PCR-amplified fragments and cloning junctions were verified by DNA sequencing. Details regarding vector construction are available upon request. For in vitro drug assays, lymphoma cells with different genotypic backgrounds were subjected to cyclophosphamide (CTX) Pude Pharm. Corp., Shanxi, China) at a concentration of $20 \mu \mathrm{M}$ for $4 \mathrm{~h}$, and viability and apoptosis were measured $4 \mathrm{~h}$ later (see following section).

Western blot analysis. Lymphoma cell and MEFs harboring variant genotypes were incubated for $24 \mathrm{~h}$ in $0.1 \%$ fetal bovine serum (FBS) (low serum), lysed using protein extraction buffer and homogenized in $250 \mu 1$ of lysis buffer. Cytoplasmic and mitochondrial extracts corresponding to $60 \mu \mathrm{g}$ of protein (BioRad Bradford protein assay) were electrophoresed through sodium dodecyl sulfate polyacrylamide gel electrophoresis (SDS-PAGE) on 4 to $20 \%$ gradient gels (Shanghai Sangon Biotech, Shanghai, China) and transferred to nylon membranes (Shanghai Sangon Biotech). The blots were probed with primary antibodies to recognize respective proteins, including anti-Bim against Bim-extra long (EL), Bim-long (L), Bim-short (S) (1:150, Santa Cruz Biotechnology, Santa Cruz, CA, USA), anti-Puma, anti-Myc (\#sc-142), anti-Bcl-2, anti-phosphorylated Bcl-2 that recognizes epitope Ser 87 (1:40, Santa Cruz Biotechnology), anti-cleaved caspase 3 (Asp175) (1:100) and anti-cleaved caspase 9 (Asp330) (1:100; Cell Signaling Technology). Horseradish peroxidase-coupled mouse anti-rabbit immunoglobulin (Jingmei Biotech Co., Ltd., Shenzhen, China) secondary antibodies were subsequently incubated for $1 \mathrm{~h}$ at room temperature. The subsequent protein was analyzed with chemiluminescence using an ECL Western blotting kit (Amersham Biosciences, IL, USA), according to the manufacturer's instructions. 
Lymphoma reconstitution and treatment. We transplanted uncultured or retrovirally infected lymphoma cells into syngeneic p53 knockdown and non-transgenic 6- to 10 -week-old C57BL/6 mice by tail vein injection $\left(10^{6}\right.$ viable lymphoma cells in PBS). Mice were fed $0.02 \mathrm{mg} / \mathrm{ml}$ tetracycline-containing drinking water every 4 days to activate the target Bim, and received a single intraperitoneal dose of $300 \mathrm{mg} / \mathrm{kg}$ body weight of CTX upon tumor manifestation. The recipient mice were monitored for tumor formation twice a week by palpation of the pre-scapular and cervical LNs. Enlargements of at least $5 \mathrm{~mm}$ in the longest diameter were considered 'well-palpable' and reflective of malignant disease. Tumor-transplanted mice were subjected to survival monitoring and histopathological evaluation, as described previously.

Assessment of viability and apoptosis. The viabilities of cultured serum starvation lymphoma cells, MEFs were measured by 3-(4,5-dimethylthiazol-2-yl)-2,5-diphenyl-tetrazolium bromide (MTT) assay using an ELISA reader (Anthos Mikrosysteme $\mathrm{GmBH}$, Germany) at a wavelength of $570 \mathrm{~nm}$. A standard optical density of the untreated control cells was considered at $100 \%$ viability to calculate the viability of the treated cells. The level of apoptosis was analyzed by a fluorescence-based terminal deoxynucleotidyl transferase dUTP nick end labeling (TUNEL) assay. The commercially available APO-BrdU ${ }^{\mathrm{TM}}$ TUNEL assay kit (with Alexa Fluor ${ }^{\circledR} 488$ anti-BrdU, 60 assays) (Invitrogen) was utilized to label DNA fragmentation with terminal deoxynucleotide transferase following the instructions of the manufacturer. The FACS apoptotic cell analysis was quantified (Calibur, BD Biosciences, CA, USA) using the geo mean function of the CellQuest Software (FACScan, BD Biosciences). All flow cytometry analyses were repeated in triplicate.

Immunofluorescence and confocal assays. For immunostaining, the cells were fixed in $4 \%(\mathrm{v} / \mathrm{v})$ paraformaldehyde for 10 min at room temperature, permeabilized in $0.1 \%$ Triton X-100 solution for $10 \mathrm{~min}$ and stained with primary anti-Bax antibodies (1:200, Santa Cruz Biotechnology) and anti-cytochrome c (1:200, Cell Signaling Technology) for $30 \mathrm{~min}$ at room temperature. Following washing with PBS, cells were incubated with affinity-purified FITC- or rhodamine-conjugated secondary antibodies (mouse anti-rabbit IgG, 1:4000, Jackson Immuno Research, West Grove, PA, USA). Following $1 \mathrm{~h}$ of incubation, slides were mounted and the stained cells were analyzed under a confocal microscope (Leica Microsystems Heidelberg $\mathrm{GmbH}$, Heidelberg, Germany) at an excitation of $488 \mathrm{~nm}$ and emission of $525 \mathrm{~nm}$.

Mouse footpad injections and in vivo imaging. Mouse footpad injections were performed as described previously. In a typical experiment, a total volume of $50 \mu \mathrm{l} \mathrm{PBS}$, containing $10 \mu \mathrm{g}$ Bim siRNA or pTHE-Bim with $10 \mu \mathrm{g}$ of expression plasmid, was intradermally injected into a mouse footpad. At the indicated points in time, the mice were imaged $10 \mathrm{~min}$ following intraperitoneal injection of luciferin $(100 \mu \mathrm{l}$ of $30 \mathrm{mg} / \mathrm{ml}$ luciferin; $150 \mathrm{mg} / \mathrm{kg}$ body weight). Mice were sedated using isoflurane and live anesthetized mice were imaged using the IVIS100 in vivo imaging system (Xenogen Corp., Alameda, CA, USA). The resulting light emission was quantified using LivingImage
A

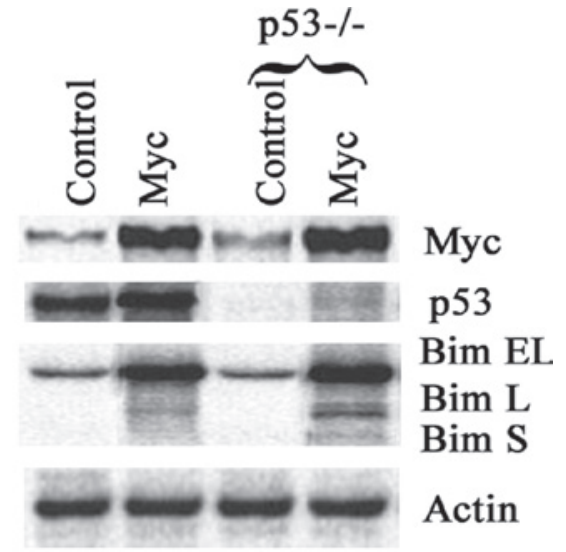

B

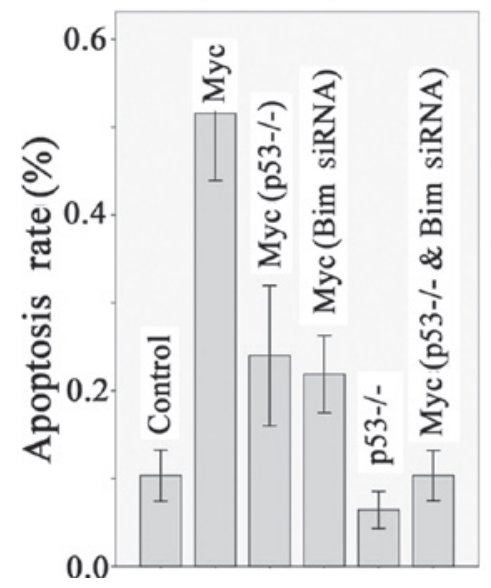

Figure 1. Resistance to Myc-induced apoptosis caused by the silencing of Bim was enhanced by concomitant p53 deficiency. (A) Bim and p53 expression in Myc-induced lymphoma cells. Cells were infected with MSCV-Myc in either $\mathrm{p} 53^{-/}$or control MEF, lysates were collected and proteins analyzed by immunoblotting. The three isoforms of BIM (Bim EL, Bim L and Bim S) are shown. (B) MEFs were either mock-transfected or transfected with negative control or MSCV-Bim for $24 \mathrm{~h}$ and followed with serum starvation. Transfected cells were then subjected to assessment of apoptosis by Annexin V/PI staining, as described in Materials and methods. Untreated cells (none) served as the control (100\%). The experiment was performed in triplicate. All of the results depicted represent a mean of at least three independent experiments. Bars represent the means \pm SD of 3-5 animals of each genotype. MSCV, murine stem cell virus; SD, standard deviation; MEFs, mouse embryo fibroblasts; siRNA, small interfering RNA; PI, propidium iodide.

software (Xenogen Corp), written as an overlay on Igor image analysis software (WaveMetrics Inc., Lake Oswego, OR, USA). Raw values are reported as photons $/ \mathrm{sec} / \mathrm{cm}^{2} / \mathrm{sr}$.

\section{Results}

Bim is critical but is not the sole BH3-only protein mediating Myc-induced apoptosis. We hypothesized that Bim plays a crucial role following deregulated expression of Myc and represent a potential novel apoptotic induction target for tumor intervention. To discriminate this and evaluate the extent of its contribution, we studied the expression of Bim in MEFs followed by Myc infection. Immunoblot analysis revealed that the levels of all three major splicing isoforms (Bim EL, Bim $\mathrm{L}$ and Bim S) were induced following Myc infection in both MEFs but not in the normal control. Moreover, Myc appeared to induce significant dephosphorylation of Bim EL (Fig. 1A). 
This finding is notable because others have shown that dephosphorylation of Bim increases its proapoptotic function (7). We further examined the proapoptotic effect of Bim through transfection of Bim in MEF cells. Myc transfection for $24 \mathrm{~h}$ in MEFs induced cell apoptosis by approximately $21 \%$; further introduction of Bim for $24 \mathrm{~h}$ enhanced the inhibitory effects by almost 39\%, as compared to the apoptosis rate (Fig. 1B). The up-regulation of Bim was not attenuated in $\mathrm{p} 53^{-/-}$MEFs following Myc infection (Fig. 1B), suggesting that p53 deficiency does not completely mask the Myc-induced apoptosis signal. When we silenced Bim in $553^{--}$MEFs, the inhibition effect attained maximum values, as indicated in Fig. 1B. By comparison, cells transfected with control siRNA against GFP continued to undergo apoptosis in response to Myc; levels were similar in cells that did not receive siRNA.

Bim-dependent apoptosis is involved in Bax/mitochondrial apoptosis signaling. Myc-transduced tissue-specific transgenic mice were identified histopathologically (Fig. 2A). Lymphoma cells were isolated and apoptosis was measured in a series of matched controls, Bim-overexpressing and silenced lymphomas cells. Cells were then subjected to serum starvation in order to trigger apoptosis. Lymphoma cells overexpressing Bim were capable of releasing cytochrome c from mitochondria, altering localization of the proapoptotic protein, Bax, and inducing caspase activation, as indicated by Western blots for caspases 3 and 9. These caspases may be blocked by the caspase inhibitor z-VAD-fmk (Fig. 2B). Using the methods of immunostaining by confocal microscopy, we found that Bax alone displayed a nuclear staining pattern in the lymphoma cells. Following transfection with Bim, Bax was found in the cytoplasm in apoptotic cells, indicating its activition by Bim. In the meantime, the distribution of cytochrome $\mathrm{c}$ changed from punctate to more diffuse, verifying the release of cytochrome $\mathrm{c}$ from mitochondria (Fig. 2C). In addition, there was some overlap observed between Bax and cytochrome c staining by merging their images. This was further verified through the measurement of cytochrome $c$ in the cytoplasm using methods previously described. These observations suggest that Bax translocates to mitochondria during Bim-induced apoptosis in lymphoma cells. In addition, concomitant with synergistic toxicity, Bim plus p53 could also increased cytochrome c release in our system more than Bim alone (Fig. 2C), indicating that cellular inhibition of Bim or p53 is primarily through cascade and mitochondrial pathways. Such a mechanism is consistent with the finding that Bim and p53 may act in concert in response to gamma-radiation and glucocorticoids (20).

Loss of Bim accelerates morbidity in mice with Myc-induced lymphoma. In order to evaluate the apoptotic effect of Bim on lymphomas in vivo, we investigated whether loss of Bim was capable of accelerating lymphoma penetrance. Firstly, we designed and produced lentiviral vectors that expressed a shRNA targeting Bim and a control sequence. An infection rate of almost $100 \%$ was achieved in the lymphomas, and the provirus vector was stably integrated as indicated by the expression of a GFP marker that was encoded by the lentivirus, and remained constant and complete in the Bim shRNA-infected lymphoma population 8 weeks following
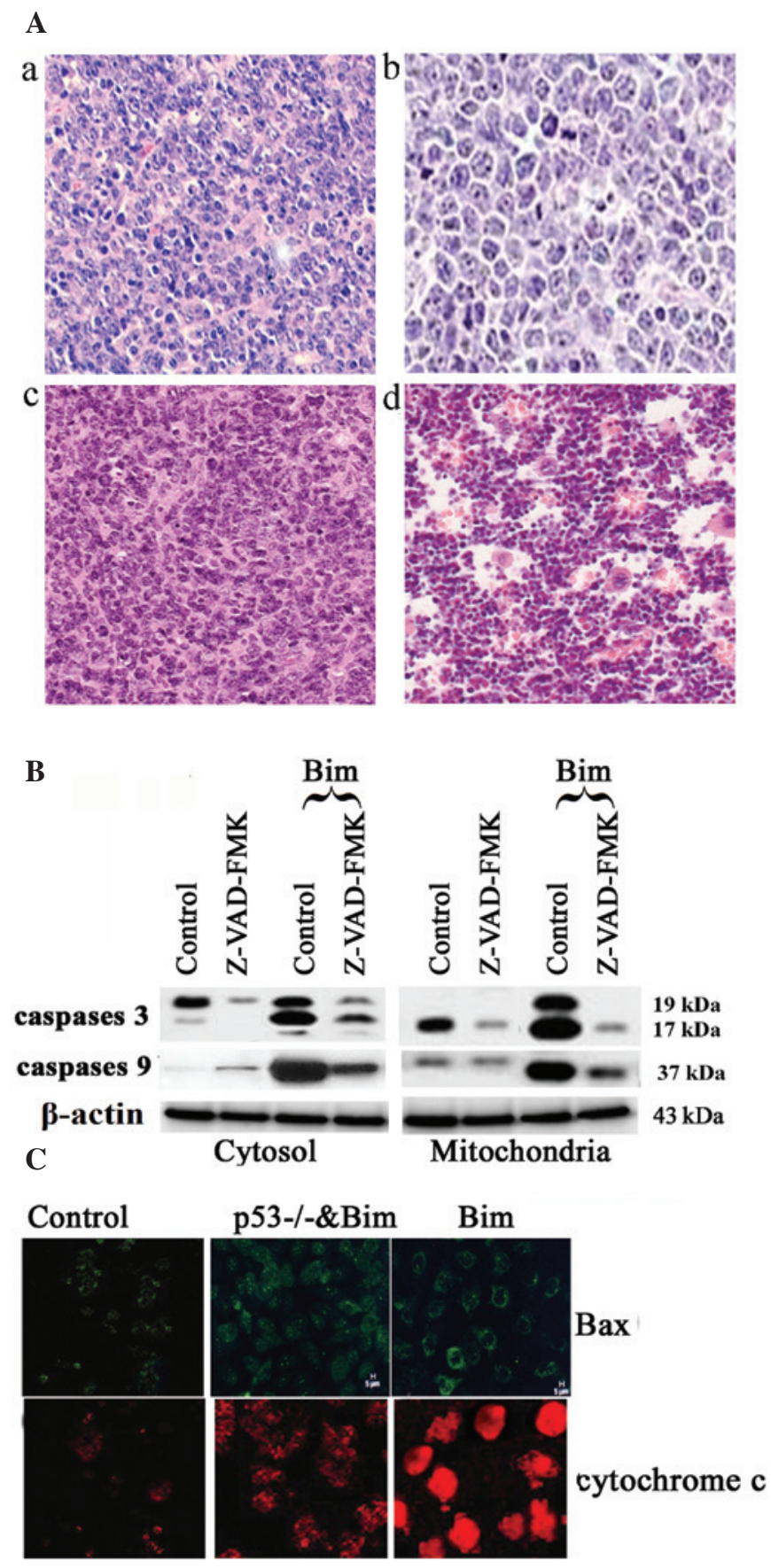

Figure 2. Bim potentially synergizes with p53 in mediating the apoptosis involved in Bax/mitochondrial apoptotic signaling. (A) H\&E staining of lymph node (a and b), splenic (c) and bone marrow (d) sections of animals transplanted with lymphoma cells lacking Bim. (a and b) Lymph node lacking Bim presented diffuse, large B-cell lymphoma. (c) Splenic involvement by diffuse, large cell lymphoma in recipient. (d) Hypercellular bone marrow with expanded peritrabecular zones containing diffuse, large cell sheaths in the recipient. (B) Either Bim-transduced or control lymphoma cells were incubated with Z-VAD-fmk and harvested to extract cytosolic and mitochondrial proteins, followed by separation by SDS-PAGE, as described in Materials and methods. Membrane blots were incubated with antibodies that recognize active caspases 3 and 9 (cleaved forms) as indicated. Images are representative of one of the three studies. Caspases 3 and 9 were activated by Bim and inhibited in the presence of $20 \mu \mathrm{M}$ Z-VAD-fmk. (C) Bim induces colocalization of Bax and cytochrome $\mathrm{c}$ in lymphoma cells undergoing apoptosis. Lymphoma cells of different genotypes, as indicated, were fixed and immunostained with antibodies against Bax (green) and cytochrome c (red), as described in 'Materials and methods'. Cells were imaged by confocal microscopy. SDS-PAGE, sodium dodecyl sulfate polyacrylamide gel electrophoresis; H\&E, hematoxylin and eosin. 
A

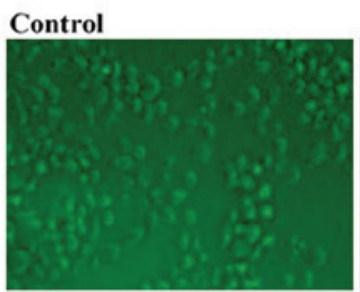

Lenti-shRNA
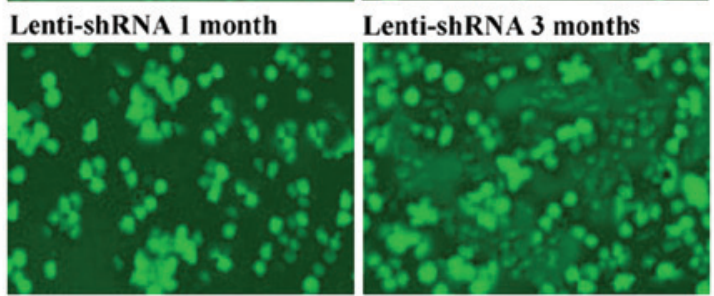

$\mathbf{C}$

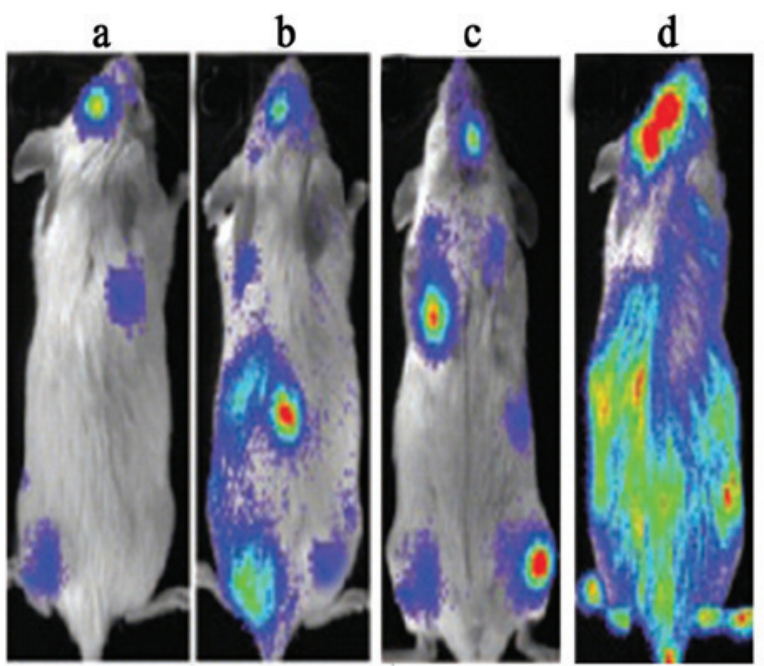

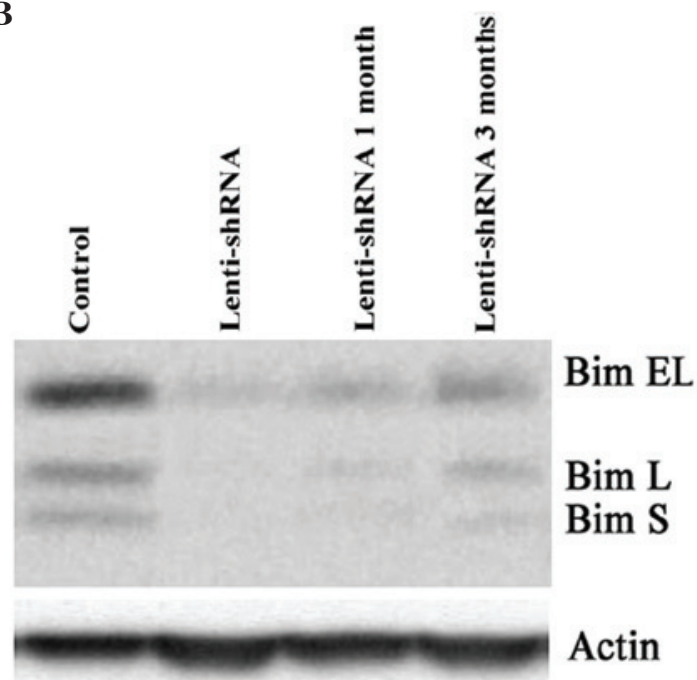

D

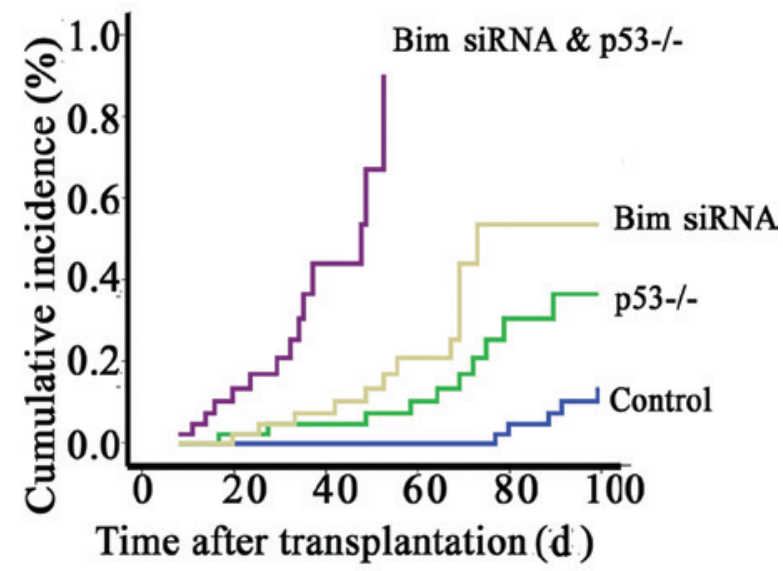

Figure 3. Bim knockdown by siRNA attenuates apoptosis induced by Myc. (A) lymphoma cells were infected with a lentivirus encoding Bim shRNA and analyzed by fluorescence microscopy for the expression of GFP. The efficiency of infection was determined by assaying GFP expression at various infection times as indicated. Stable GFP expression in lymphoma cells was determined 3 months later compared with uninfected controls. (B) Stable down-regulation of Bim by lentivirus-delivered shRNA. Representative bands of Western blots revealed considerable decreases in Bim levels relative to control lymphoma cells until 3 months following infection with lentivirus, while displaying no changes in the levels of a control protein, actin. (C) Whole-body fluorescence imaging allows the visualization of lymphoma dissemination with the indicated genotypes 30 days after lymphoma transplantation. Luciferase expression was determined following intraperitoneal luciferin injection by whole-animal imaging using the Xenogen IVIS200 in vivo system. (d) Both p53 and Bim deficiency lymphomas are much more disseminated, infiltrating liver, kidneys, lung and brain, while (a) the control, (b) Bim-silenced and (c) p53 $3^{-/}$lymphoma was restricted to the lymphoid compartment. Fluorescent images are representative examples from several independent experiments per genotype. (D) Cumulative incidence of lymphoma in mice of the indicated genotype. Both the silencing of Bim and p53 loss accelerated lymphoma development initiated by the Myc oncogene (P<0.0001, log-rank test). siRNA, small interfering RNA; shRNA, short hairpin RNA; GFP, green fluorescent protein.

infection (Fig. 3A). Western blot analysis revealed effective Bim silencing (the reduction in the expression of Bim) in both the basal state and when lymphomas were transplanted into recipient mice (Fig. 3B). Consistent with the histopathological findings, whole-body fluorescence imaging, with more dissemination, was used to observe an aggressive invasive growth pattern, including the liver, kidneys, lung and brain in Bim knockdown and p53-null lymphomas. Tumor cells were restricted to the LN, bone marrow and spleen in controls, despite a similarly large tumor burden (Fig. 3C). Control lymphoma cells developed a follicular lymphoma stochastically when transplanted into recipient mice at a low penetrance following a long latency period of $>3$ months (the latency was $66 \pm 21$ days); only 5 of the 15 monitored mice developed a tumor. However, when Bim deficiency was present we observed a striking acceleration of penetrance: 13 out of the 15 observed mice developed tumors $(\mathrm{P}<0.005$ for Bim loss vs. control) and exhibited a significantly reduced latency (38 \pm 16 days). Mice with Bim knockdown that were p53-null formed tumors relatively rapidly, with an identical high penetrance (15 out of 15 mice developed tumors) and a short latency (33 \pm 18 days) (Fig. 3D). As expected, mice harboring both Bim knockdown and p53-null lymphoma displayed decreased survival time, with tumor cells that disseminated frequently and extensively into non-lymphoid organs. 
A

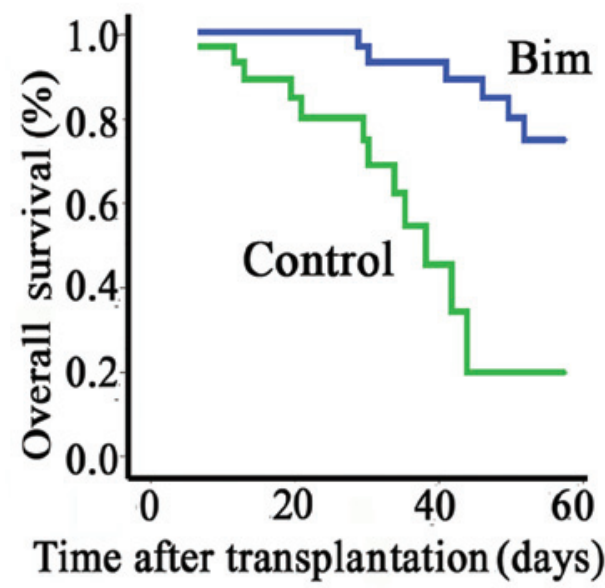

B

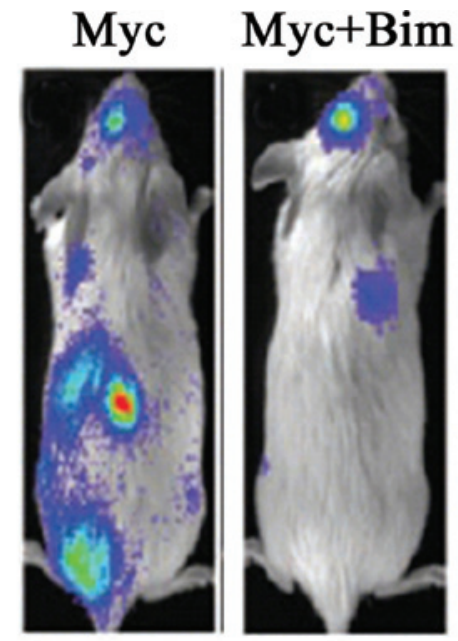

C
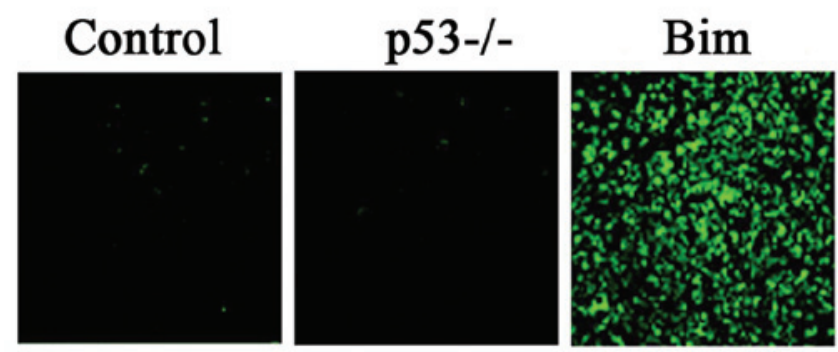

p53-/- \& Bim
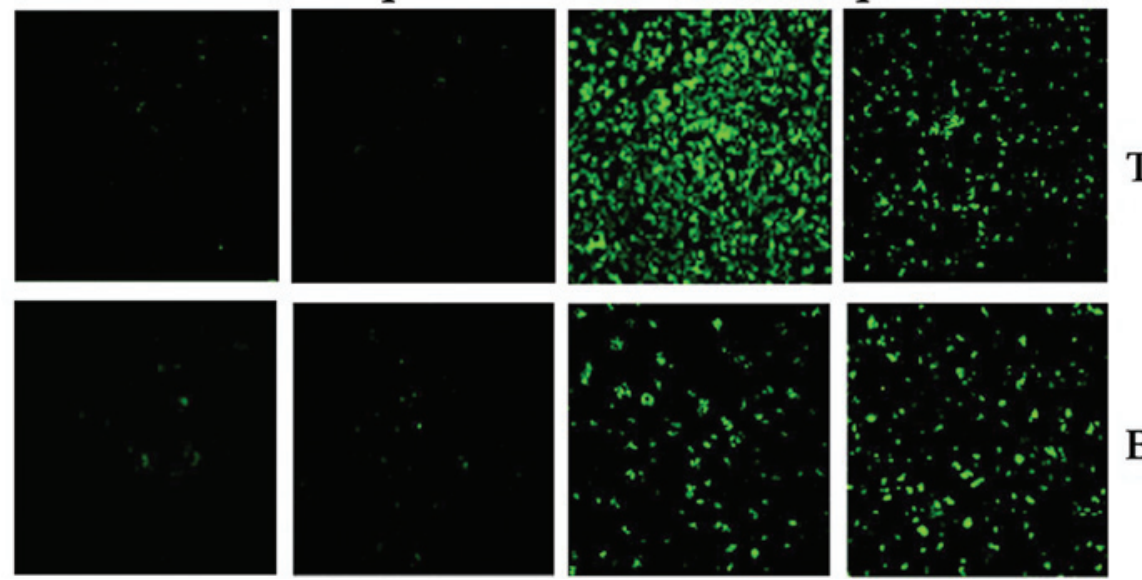

TUNEL

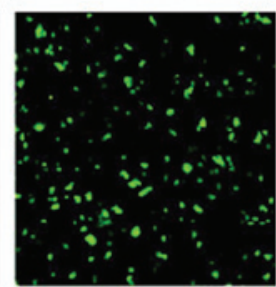

Figure 4. Bim-enhanced survival of mice loaded with lymphoma. (A) Survival of mice harboring Bim overexpression and controls were plotted in a Kaplan-Meier survival curve format. (B) Whole-body fluorescence imaging demonstrated that Myc lymphomas were much more disseminated than the Bim-overexpressing lymphoma 60 days following lymphoma transplantation. (C) Apoptosis in situ (lymph nodes) was visualized by TUNEL; positive fluorescence reveals the in situ distribution of Bim expression in the same tissue. Bim-overexpressing lymphomas displayed significantly more apoptosis compared to the controls. TUNEL, terminal deoxynucleotidyl transferase dUTP nick end labeling.

Bim is capable of prolonging the survival of lymphomabearing mice. We next measured the ability of Bim to prolong the survival of lymphoma-bearing mice. We transplanted pTHE-Bim-infected and uninfected lymphoma cells derived from the same primary lymphoma into recipients, fed the mice with tetracycline-containing drinking water to trigger Bim expression and then monitored the palpable lymphoma masses twice a week. Following transplantation, $48 \%$ of the Bim-overexpressing lymphomas failed to present palpable LNs during the period of observation. The median survival of Bim-transfected mice was significantly extended compared with uninfected, directly transplanted counterparts (86 \pm 39 days for Bim transduction and $32 \pm 11$ days for control) (Fig. 4A, P=0.005). Lymphoma restriction followed by Bim overexpression was further visualized using whole-body fluorescence imaging (Fig. 4B), which indicated that lymphoma inhibition was driven by the introduced proapoptotic Bim gene. Moreover, autopsy of vehicle-treated mice revealed disseminated disease throughout the lymphoid system with enlarged LNs and splenomegaly. Bim-overexpessing mice autopsied at the same time and assessed by TUNEL assay displayed a dramatic increase in apoptotic cells (Fig. 4C).

Bim has a profound effect on drug-induced cell death in vitro and in vivo. The $\mathrm{p} 53^{-/}$mice were used as recipients to generate Myc-induced lymphomas. The isolated lymphoma cells were then subjected to Bim siRNA to establish a cohort of lymphoma cells containing Bim and p53 double-deficient plus all relevant controls. The cells were then challenged with various cytotoxic agents, respectively, in particular with CTX. Cell apoptosis was monitored over a period of $72 \mathrm{~h}$ by means of flow cytometric analysis with Annexin V/propidium iodide (PI) staining. Loss of either Bim or p53 had a profound effect on drug-induced cell death in vitro. Lymphoma cells lacking both Bim and p53 and treated with CTX survived significantly longer than cells lacking only Bim or p53 and, of course, wild-type (WT) cells. In both $\mathrm{Bim}^{-/}$and $\mathrm{p} 53^{-/-}$cells, apoptosis was almost undetectable; control cells displayed almost a 6- to 10-fold accumulation of apoptotic cells. Lymphomas lacking only Bim or p53 displayed moderate apoptosis 


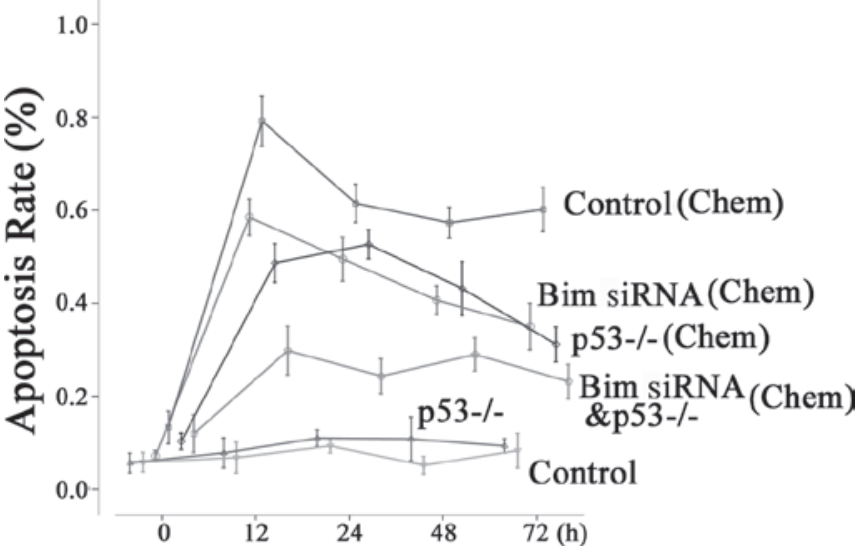

Figure 5. Synergy between Bim knockdown and p53 deficiency protects lymphoma cells against CTX-induced killing. Parental (including $\mathrm{p} 53^{-/}$or control) and Bim RNAi knockdown subclones of lymphoma cells were treated for $24 \mathrm{~h}$ with DMSO (vehicle control; NT) or $1 \mu \mathrm{M} \mathrm{CTX}$ and cell death was assessed as indicated above. Data represent the means \pm SEM of three experiments indicating percentages of cell death compared to untreated cells. Cells lacking Bim showed resistance to CTX chemotherapy $(\mathrm{P}<0.01)$. CTX, cyclophosphamide; DMSO, dimethyl sulfoxide; SEM, standard error of the mean.

(Fig. 5). Expression of Bim in $\mathrm{p} 53^{-/-}$cells partly restored the susceptibility of these cells to the chemotherapy agent CTX as described previously. This indicates that Bim and p53 have overlapping roles in the chemotherapy agent-induced killing of lymphoma cells. Thus, we can conclude that Bim loss has a potent anti-apoptotic effect following treatment in isolated lymphoma cells.

\section{Discussion}

In this study, by comparing the properties of Myc-induced lymphomas from a number of genetic backgrounds, we extended the observation of the proapoptotic Bcl-2 family member, Bim, a major regulator of lymphoid and myeloid homeostasis and showed that Bim is a key apoptotic effector of Myc in sensitizing cells with Myc-induced cell apoptosis. Bim deletions may impact tumor development and anticancer therapy. In addition, activation of Bim is essential for tumor cell killing and critical for the Myc-Bcl-2 signaling cascade. Furthermore, we demonstrated that cooperative effects between p53 and Bim loss contribute to the marked Myc sensitivity of lymphomagenesis.

Bim has been proven to be a proapoptotic target of Myc, and loss of p53 is unable to suppress Bim expression in certain cell types. In this report, Myc was capable of inducing the proapoptotic $\mathrm{BH} 3$ protein, Bim, while deficient Bim activity was able to impair Myc-mediated apoptosis. Bim overexpression resulted in enhanced cell death and growth inhibition in response to the Myc oncogene, suggesting that Bim is essential for the initiation of Myc-induced apoptosis. Similar dependence on Bim for apoptosis by other cancer cells has also been observed (15-17). Although the elevated level of Bim in MEF cells suggests that Bim is a proapoptotic target of Myc, we have, until now, been unable to demonstrate any direct activation of Bim by Myc.
This raises the possibility that the loss of Bim consequently accelerates Myc-induced lymphomagenesis. To confirm and extend the effects of Bim to Myc-induced lymphoma, we examined the impact of Bim expression or silencing on Myc-induced lymphoma development or apoptosis of isolated lymphoma cells following short-term culture through a system for rapidly generating tissue-specific transgenic mice. Such animals develop B-cell lymphomas a number of months later. This model provided a candidate screen to identify the apoptotic proteins. First, various combinations of transgenes or stem cell genotypes may be easily created, providing a relevant setting for rapidly comparing the impact of Myc and Bim on tumor behavior. Second, all of the altered genes are capable of being easily monitored, a property that facilitates examining signal transduction for apoptosis. In addition, essentially pure tumor cells may be isolated from LNs and studied ex vivo by retrovirally transducing primary lymphomas to overexpress a gene of interest. These cells may also be expanded in genetically matched nontransgenic recipients $(18,19)$.

In order to generate a cohort of lymphoma cells containing Bim and p53 double-deficiency in vivo, viable selected stable Bim-silenced lymphoma cells were immediately transplanted into the tail vein of syngeneic mice recipients, wherein they were allowed to expand in vivo. Endogenous Bim expression was confirmed to be attenuated with Bim siRNA, while Bim was unaffected in control mice. This method provided a rapid alternative for generating complex tumor genotypes. These lymphomas were histopathologically identical to their respective primary tumors when aliquots of the same population were propagated in separate recipients $(21,27)$.

Either Bim or p53 loss in lymphomas appears to be indispensable for the aggressive behavior of lymphoma. Further evidence obtained through TUNEL assay in the current study indicated that apoptosis was involved in Bim-induced cell death, independent of $\mathrm{p} 53$. We also observed that the up-regulation of Bim determines the sensitivity of lymphoma cells to the apoptotic effects of certain chemotherapy agents. Although it has been previously indicated that p53 is capable of cooperating with Bim to play a role in apoptosis induction (26), the data presented herein provide compelling evidence that, while p53 induction is required for Myc-induced apoptosis, it may not be sufficient to provoke cell death and that Bim is essential for the apoptosis elicited in lymphoma cells harboring Myc activation. Indeed, this study provides formal genetic proof that Bim and p53 may present different targets of Myc. Similarly, Bim may reside in a position in the pathway that does not influence p53, Bim and p53 must function in separate pathways. Therefore, although Bim and p53 may function in the same tumorsuppressor pathway of Myc-Bax, our results demonstrate that this pathway must bifurcate. There is cooperativity between Bim and p53 at least at some level. Understanding how the involved signaling components work would provide critical information to help design strategies to augment their efficacy.

One of the most intriguing outcomes of this study is that we confirmed and extended the signal network by linking Myc with p53 and Bim. There is a cooperative effect on the rate of tumorigenesis in mice lacking p53 with silenced Bim in comparison to mice deficient in $\mathrm{p} 53$ alone. This implies that Bim does synergize the p53 functions involved in the proliferation ability of lymphoma, although we have not 
yet analyzed the secondary changes of Bim and p53 loss in detail. In this context, it could be possible that the enhancement of Bim and p53 expression or simultaneous activation of downstream targets might be a promising strategy for the treatment of lymphoma, particularly in the context of conventional theraputic resistance. Conventional therapies mainly rely on activating the cell death program indirectly and it seems likely that strategies that could directly activate cell-suicide machinery would be more effective (22). It has been verified that BH3 mimetics, such as ABT-737, substantially enhanced apoptosis in a number of types of the tested cells $(23,24)$. The search for BH3 mimetics specifically targeted to both Bim and p53 signals is intensifying and some promising leads are beginning to emerge.

\section{Acknowledgements}

We thank Professor Wang Wei for providing technical assistance and insightful discussions during the preparation of the manuscript. We gratefully acknowledge Professor Scott W. Lowe (Howard Hughes Medical Institute, Cold Spring Harbor, New York, NY, USA) for providing retroviral vectors. We thank Dr Xiaoyong Zhang at the Wistar Institute, USA, for help with linguistic revision of the manuscript. The research was supported by the National Natural Science Foundation of China (nos. 30973440, 30770950), the Key Project of Shandong Scientific and Technology (2008GG30002034), and the key project of Chongqing Natural Science Foundation (CSTC, 2008BA0021).

\section{References}

1. Hemann MT, Bric A, Teruya-Feldstein J, Herbst A Nilsson JA, Cordon-Cardo C, Cleveland JL, Tansey WP and Lowe SW: Evasion of the p53 tumour surveillance network by tumour-derived MYC mutants. Nature 436: 807-811, 2005.

2. You Z, Madrid LV, Saims D, Sedivy J and Wang CY: c-Myc sensitizes cells to tumor necrosis factor-mediated apoptosis by inhibiting nuclear factor $\kappa \mathrm{B}$ transactivation. J Biol Chem 277: 36671-36677, 2002.

3. Ravitz MJ, Chen L, Lynch M and Schmidt EV: c-myc repression of TSC 2 contributes to control of translation initiation and Myc-induced transformation. Cancer Res 67: 11209-11217, 2007.

4. Adachi S, Obaya AJ, Han Z, Ramos-Desimone N, Wyche JH and Sedivy JM: c-Myc is necessary for DNA damage-induced apoptosis in the G(2) phase of the cell cycle. Mol Cell Biol 21: 4929-4937, 2001

5. Prendergast GC: Mechanisms of apoptosis by c-Myc. Oncogene 18: 2967-2987, 1999.

6. Smith DP, Bath ML, Metcalf D, Harris AW and Cory S: MYC levels govern hematopoietic tumor type and latency in transgenic mice. Blood 108: 653-661, 2006.

7. Egle A, Harris AW, Bouillet P and Cory S: Bim is a suppressor of Myc-induced mouse B cell leukemia. Proc Natl Acad Sci USA 101: 6164-6169, 2004.

8. Eischen CM, Weber JD, Roussel MF, Sherr CJ and Cleveland JL: Disruption of the ARF-Mdm2-p53 tumor suppressor pathway in Myc-induced lymphomagenesis. Genes Dev 13: 2658-2669, 1999.

9. Sharma SV, Gajowniczek P, Way IP, Lee DY, Jiang J, Yuza Y, Classon M, Haber DA and Settleman J: A common signaling cascade may underlie 'addiction' to the Src, BCR-ABL, and EGF receptor oncogenes. Cancer Cell 10: 425-435, 2006.
10. Vervoorts J, Lüscher-Firzlaff $\mathbf{J}$ and Lüscher B: The ins and outs of MYC regulation by posttranslational mechanisms. J Biol Chem 281: 34725-34729, 2006.

11. Dansen TB, Whitfield J, Rostker F, Brown-Swigart L and Evan GI: Specific requirement for Bax, not Bak, in Myc-induced apoptosis and tumor suppression in vivo. J Biol Chem 281: 10890-10895, 2006.

12. Payne SR and Kemp CJ: Tumor suppressor genetics. Carcinogenesis 26: 2031-2045, 2005.

13. Slack A, Chen Z, Tonelli R, Pule M, Hunt L, Pession A and Shohet JM: The p53 regulatory gene MDM2 is a direct transcriptional target of MYCN in neuroblastoma. Proc Natl Acad Sci USA 102: 731-736, 2005.

14. Yu D and Thomas-Tikhonenko A: A non-transgenic mouse model for B-cell lymphoma: in vivo infection of p53-null bone marrow progenitors by a Myc retrovirus is sufficient for tumorigenesis. Oncogene 21: 1922-1927, 2002.

15. Cragg MS, Kuroda J, Puthalakath H, Huang DC and Strasser A: Gefitinib-induced death of NSCLC cell lines expressing mutant EGFR requires BIM and can be enhanced by BH3 mimetics. PLoS Med 4: 1681-1689, 2007.

16. Naik E, Michalak EM, Villunger A, Adams JM and Strasser A: Ultraviolet radiation triggers apoptosis of fibroblasts and skin keratinocytes mainly via the BH3-only protein. J Cell Biol 176: 415-424, 2007.

17. Akiyama T, Bouillet P, Miyazaki T, et al: Regulation of osteoclast apoptosis by ubiquitylation of proapoptotic BH3-only Bcl-2 family member Bim. EMBO J 22: 6653-6664, 2003.

18. Schmitt CA, Rosenthal CT and Lowe SW: Genetic analysis of chemoresistance in primary murine lymphomas. Nat Med 6: 1029-1035, 2000.

19. Schmitt CA, McCurrach ME, de Stanchina E, Wallace-Brodeur RR and Lowe SW: INK4a/ARF mutations accelerate lymphomagenesis and promote chemoresistance by disabling p53. Genes Dev 13: 2670-2677, 1999.

20. Erlacher M, Michalak EM, Kelly PN, Labi V, Niederegger H, Coultas L, Adams JM, Strasser A and Villunger A: BH3-only proteins Puma and Bim are rate-limiting for gamma-radiationand glucocorticoid-induced apoptosis of lymphoid cells in vivo. Blood 106: 4131-4138, 2005.

21. Schmitt CA and Lowe SW: Apoptosis and chemoresistance in transgenic cancer models. J Mol Med 80: 137-146, 2002.

22. Chauhan D, Velankar M, Brahmandam M, Hideshima T, Podar K, Richardson P, Schlossman R, Ghobrial I, Raje N, Munshi $\mathrm{N}$ and Anderson KC: A novel Bcl-2/Bcl-X(L)/ Bcl-w inhibitor ABT-737 as therapy in multiple myeloma. Oncogene 26: 2374-2380, 2007.

23. Del Gaizo Moore V, Schlis KD, Sallan SE, Armstrong SA and Letai A: BCL-2 dependence and ABT-737 sensitivity in acute lymphoblastic leukemia. Blood 111: 2300-2309, 2008.

24. Witham J, Valenti MR, De-Haven-Brandon AK, Vidot S, Eccles SA, Kaye SB and Richardson A: The Bcl-2/Bcl-XL family inhibitor ABT-737 sensitizes ovarian cancer cells to carboplatin. Clin Cancer Res 13: 7191-7198, 2007.

25. Guo CB, Li YC and Jin XQ: Chemoprotection effect of retroviral vector encoding multidrug resistance 1 gene to allow intensified chemotherapy in vivo. Cancer Chemother Pharmacol 58: 40-49, 2006.

26. Erlacher M, Labi V, Manzl C, Böck G, Tzankov A, Häcker G, Michalak E, Strasser A and Villunger A: Puma cooperates with Bim, the rate-limiting $\mathrm{BH} 3$-only protein in cell death during lymphocyte development, in apoptosis induction. J Exp Med 203: 2939-2951, 2006.

27. Lindemann RK, Newbold A, Whitecross KF, et al: Analysis of the apoptotic and therapeutic activities of histone deacetylase inhibitors by using a mouse model of B cell lymphoma. Proc Natl Acad Sci USA 104: 8071-8076, 2007. 